En descenso de lo global a lo local: Una perspectiva desde la región atlántica de Canadá

Education, social justice and place:

A multi-scalar view from Canada

\title{
Michael Corbett
}

Universidad de Acadia

michael.corbett@acadiau.ca

Recibido 10.6.2013

Revisado 12.7.2013

Aprobado 27.9.2013 


\section{Resumen $^{1}$}

Mientras la educación ha sido, por muchos años, un proyecto estatal, su implementación se ha centrado en la escala local o regional (estatal y provincial) en Norteamérica. Tendencias recientes que resaltan la globalización del discurso educativo han generado tensiones en la implementación y análisis de la educación a nivel mundial. La aparición de una medida global, como PISA y TIMSS, permite comparar distintos sistemas educativos y la creación de un ámbito educativo transnacional.

Este artículo observa una micro geografía educacional particular de tres escuelas en la región atlántica de Canadá. A partir de ello, evidencia que la data nacional presenta una limitación para la descripción, el soporte y el análisis de la performance educacional o para entender la complejidad social en lugares concretos. La ironía radica en que este nivel macro de análisis es el que conduce la política educacional, que es experimentada fundamentalmente a escala local. En el caso de zonas que se encuentran económicamente en desventaja, como la región atlántica de Canadá, un bajo rendimiento es predeciblemente crónico. Frente a ello, considero que mayor atención en una política y práctica educativas concretas y locales es necesaria tanto para el éxito de los estudiantes como para vínculos más productivos entre desarrollo económico y educación.

Palabras clave: Políticas educativas locales, macro y micro geografías, medición educativa globalizada

\section{Abstract}

While education has for many years been a state project, its governance has tended to be located at the local or regional (state and provincial) level in North America. Recent trends signalling the globalization of educational discourse have generated tensions in educational governance and analysis around the world. The emergence of global metrics such as the PISA and TIMSS allow for the comparison of different educational systems and the creation of a transnational educational arena. This paper looks at a particular educational micro geography of three schools in Atlantic Canada and argues that national data do little to describe, support or analyze educational performance or to understand social complexity in real places. The irony is

1 Este manuscrito se ha basado en la presentación del simposio WERA, presentado ante la Sociedad de Investigación Educativa Peruana (SIEP) en Lima-Perú el 15 de setiembre de 2012. 
that these macro level analyses end up driving educational policy which is experienced fundamentally at the local level. In the case of the economically disadvantaged region like Atlantic Canada, underperformance is predictably chronic. I suggest that more attention to place-sensitive educational policy and practice is needed both for student success and for more productive linkages between economic development and education.

Keywords: Place-sensitive educational policy, macro and micro geographies, educational global metrics 


\section{Macrogeografías de la educación: Medición educativa globalizada}

D urante las últimas décadas se ha creado un enérgico espacio discursivo sobre la educación global. Este nuevo espacio ha sido promovido por amplios conceptos de responsabilidad y medición y comparación de los logros educativos, que van desde el nivel de niños individuales hasta el nivel de sistemas educativos nacionales (Meyer y Benevot, 2013). En este discurso sumamente consecuente, se han creado nuevas geografías de gobernanza y análisis educativos, y ahora podemos hablar en términos comparativos y evaluativos gracias al mecanismo de medición educativa masiva como PISA (Programa para la Evaluación Internacional de Alumnos) y TIMSS (Estudio Internacional de Tendencias en Matemáticas y Ciencias).

De hecho, ha habido un discurso complementario que relaciona la mayor estandarización, las pruebas y la vigilancia general dentro de los sistemas educativos no solo con un mejor desempeño educativo, sino también con mayores medidas de justicia social a través de la responsabilidad y un análisis más sensible de las geografías sociales de bajo rendimiento educativo. La presión influyente sobre la educación y el respaldo de grandes filántropos como Bill y Melinda Gates (Kovacs, 2010), que se han interesado en el mejoramiento de las escuelas, procede del análisis que se ha realizado en esta macrogeografía y que ha "descendido" a otros lugares específicos de bajo rendimiento. Esto podría recibir el nombre de globalización descendente.

Las políticas y los temas de gobernanza educativa también han recibido una gran influencia de la emergencia -e incluso se podría decir de la hegemonía- de este espacio global emergente (Rizvi y Lingard, 2010; Ball, 2012). La noción establece que el bajo rendimiento nacional puede prestarse (Halpin y Troyna, 1995; Lingard, 2010) o aprender de las naciones con buenos resultados, una idea que ignora la cómo los sistemas educativos nacionales reflejan las culturas, sociedades y economías de las que forman parte (Luke, 2012). La educación peruana, por ejemplo, ya no puede «prestarse» del desempeño educativo de Finlandia o Singapur solo a través de políticas educativas, al punto de esperar que sus niños - por arte de magiase conviertan en pequeños finlandeses o singapurenses con el toque de una medida administrativa.

Sin embargo, al mismo tiempo, hay un discurso paralelo sobre la descentralización, expectativas de la educación con respuestas a necesidades locales, sensibilidad cultural, diferencias y temas de flexibilidad, consumismo educativo y elección de los programas educativos impartidos. Además de las tendencias homogeneizadoras, la globalización también ha creado las condiciones para compartir de una manera sin precedentes las particularidades 
de la diferencia en vastas áreas geográficas (Appadurai, 1996; Castells, 2009; Spring, 2008). A esto lo llamaré globalización ascendente.

Esto puede parecer contradictorio, pero, en el mundo de la educación actual, estos dos aliados se complementan a la perfección. De hecho, uno de nuestros más grandes retos en la educación actual es teorizar y trabajar dentro de las tensiones que emanan de la expansión y compresión simultáneas del espaco (Castells, 2000; Giddens, 1979, 1990). Estas tecnologías funcionan entre un sinnúmero de mecanismos técnicos, transaccionales y de gobernanza que simultáneamente dejan margen para las visualizaciones espaciales que van del microcronotopo hasta el global. En estas nuevas geografías, la transmisión, por ejemplo, de un video en tiempo real desde un teléfono celular económico puede mostrar lo que sucede en un rincón de un aula de clases en un momento dado en cualquier parte del mundo. Al mismo tiempo, una tabla de PISA o TIMSS puede comprimir el rendimiento educativo de una nación entera en un solo valor, un punto de datos mapeado en un cuadro comparativo global.

Sin embargo, ¿qué significan estos puntos de datos, además de que existen diferencias entre las naciones capitalistas avanzadas y de hecho muy profundas diferencias entre estas y las economías del sur global? A pesar de la emergencia de estas nuevas geografías macroeducativas, hasta ahora, tenemos una dirección global transnacional o incluso un programa curricular reducidos. De modo que el doble problema de la comparación de naciones en diferentes niveles de lo que podría llamarse "desarrollo» (grandes diferencias de recursos, distribución de dichos recursos e infraestructura educativa) y los diferentes programas curriculares abarca la idea de que las comparaciones son justas o significativas. Estos datos causan muy poca sorpresa. Desde mi perspectiva, demuestran tres aspectos fundamentales: 1) la riqueza de las naciones se refleja en los resultados educativos; 2) el logro educativo equitativamente distribuido normalmente está asociado a la orientación social colectivista a nivel del estado nacional; 3) la redistribución social de la riqueza a través de los programas sociales se asocia con un logro mayor y más equitativo.

Si bien estos resultados son bastante predecibles, en el análisis actual de las políticas educativas, existe una creciente tendencia a confiar en los datos provenientes de las evaluaciones internacionales y nacionales estandarizadas. Ello se realiza para informar y elevar el programa curricular e, incluso, la pedagogía misma en contextos como el de Canadá y los Estados Unidos, donde la dirección educativa se ha descentralizado a nivel estatal, provincial y distrital. Con estos datos, es posible imaginar escuelas que representan esencialmente lo que son, simples puntos de datos en una matriz computacional. 
Sin embargo, debo aquí resaltar que estos datos nos dicen muy poco acerca de cómo mejorar las escuelas dispares y locales que representan a las microgeografías y que deben ser comprendidas para dar paso a un cambio sensato. Los macrodatos cuantitativos estandarizados necesitan ser comprendidos y evaluados en conjunto con los microdatos exhaustivos de alcance local, que inevitablemente nos dicen más sobre cómo mejorar realmente las escuelas. La subordinación y marginalización de los enfoques cualitativos es una forma poderosa de violencia simbólica (Bourdieu, 1984) en el análisis actual de la educación.

\section{Mesogeografías: Naciones y regiones}

Las diferencias en el logro educativo a nivel internacional también son reproducidas de manera efectiva a nivel del Estado nacional. Dentro de las naciones, existen diferencias por más sorprendentes de tipo regional, rural-urbano y vecinal en los resultados educativos que subyacen a los resultados de PISA a escala nacional. Canadá, por ejemplo, cuenta con trece jurisdicciones educativas individuales, carece de un ministerio de educación y tiene una amplia autonomía local y regional de la dirección educativa, que genera una considerable diversidad interna en términos de programa curricular, pedagogía y evaluación. Estas diferencias también reflejan grandes disparidades entre las provincias en términos de ingresos familiares, educación, lengua materna y otras variables sociales, culturales y económicas que influyen en el logro educativo moderado. Sin embargo, además de estas diferencias interprovinciales/territoriales, existen importantes divergencias al interior de cada una de las propias provincias.

Aquí, comparto algunas observaciones como investigador cualitativo de la educación, cuyo trabajo se sitúa principalmente en la significativamente rural y relativamente desfavorecida región de las provincias atlánticas de Canadá. Dicho trabajo aborda la forma como el lugar y el espacio son importantes consideraciones para el análisis educativo distorsionado, siempre que la dirección educativa y el desarrollo de políticas estén protegidos por geografías educativas más amplias. Irónicamente, los temas de justicia social (p.ej., los efectos educativos de las clases sociales, la racialización, la explotación económica histórica, la discriminación de género, etc.) son a menudo presentados como problemas educativos persistentes que solo pueden ser solucionados mediante una respuesta estandarizada proveniente del Estado y fuertemente dependiente del análisis cuantitativo, como las estrategias genéricas perseguidas y respaldadas por la Fundación Gates. Estos llamados 
análisis basados en la evidencia o los datos asumen un enfoque descendente (o de arriba hacia abajo) del problema del logro educativo, que se define como el fracaso de ciertos individuos y lugares marginalizados para «tomar medidas» a un macronivel nacional e internacional. Al mismo tiempo, sin embargo, las soluciones para este bajo rendimiento son pocas veces presentadas a escala internacional o nacional, o en términos de intervenciones económicas o estructurales, tales como los pagos de la deuda externa o los esquemas de condonación, una guardería nacional o internacional o estrategias contra la violencia de género o proyectos de ingresos garantizados. Son en realidad los proveedores de servicio local los que reciben la presión de mejorar las mediciones a nivel macro, normalmente a través de pedagogías descontextualizadas y estandarizadas.

Las implicancias políticas de tal razonamiento conllevan a una estandarización y reducción cuantitativa que amplían y reducen la importancia de las microgeografías y la gobernanza local. Las microgeografías (escuelas y distritos escolares específicos) son importantes principalmente en términos de lo bien que se miden como puntos de datos en los grandes mapas de logros. Se trata de los puntos de presión de la "comunidad» donde ahora se ejerce la gobernanza (Rose, 1999), puesto que los datos comparativos son usados para «nombrar y avergonzar» a los agentes locales (p.ej., educadores, padres y alumnos) que presuntamente son de "bajo rendimiento" respecto a indicadores nacionales o niños normales según las estadísticas. A partir de esto, no se necesita examinar las mediciones o las condiciones sociales de quienes tienen un «bajo rendimiento». El único problema se sigue concentrando en saber cómo disciplinar a los rezagados y «cerrar la brecha» entre ellos y la población «normal» (Martino y Rezai-Rashti, 2013; Milner, 2013). Cuando las escuelas, regiones e incluso naciones sean reducidas a puntos de datos en una matriz comparativa, la realidad de la pedagogía, el programa curricular y el contexto se habrán perdido. Si bien la globalización y las fuerzas de cambio económico y social implicadas han creado estas nuevas geografías, al mismo tiempo, han generado condiciones para un mayor énfasis a nivel local y sobre la diversidad, la diferencia, la particularidad y, más aún, el consumidor mismo.

\section{Microgeografías: Tres aulas de clase}

Lo que la investigación en las escuelas ha demostrado es el hecho de que los factores contextuales conectados a la asistencia de los niños a las escuelas parecen hacer una gran diferencia en el logro académico. De hecho, el aula de clase mismo es un contexto social para la vida y el aprendizaje de los 
niños, y abarca también un contexto más amplio de vecindad, comunidad, provincia/estado, región y nación. Para pensar en justicia social en la educación, tiene sentido pensar cuidadosamente en escalas, como bien lo indica Nespor (2004), en un intento por comprender la interacción entre el aula de clases y un ambiente más amplio. Para plantear el asunto de manera concreta, observaremos tres aulas de clase de las provincias atlánticas de Canadá en tres escuelas que se encuentran en diferentes situaciones. Cada una de esas escuelas representa una ventana etnográfica para la práctica educativa en escuelas específicas que aparecen como puntos de datos en las evaluaciones educativas descendentes, que van desde pruebas estandarizadas a nivel provincial hasta evaluaciones de habilidades a escala nacional e internacional (como PISA y TIMSS).

Las siguientes descripciones han sido tomadas de los diarios de campo que he mantenido por un período de cinco años al realizar tres estudios diferentes de aprendizaje del lenguaje y tecnologías educativas en escuelas de las provincias atlánticas de Canadá. Cada una de estas escuelas ha recibido el calificativo de "excelente» en su propia comunidad por razones que difieren entre un lugar y otro. Cada escuela, sin embargo, se desarrolla de manera diferente en las evaluaciones estandarizadas.

\section{Urbanidad y orden}

La primera escuela es urbana y alberga a aproximadamente doscientos niños en edad de educación primaria. La parte original de su estructura se remonta a la década de los treinta y se aprecian varias adiciones -con el transcurrir de los años- a la estructura original. Las partes más modernas de la escuela contrastan su arquitectura de ladrillos del vecindario frente a la construcción comercial y residencial cercana. El complejo original de la escuela no combina tan bien con el paisaje y representa más bien una majestuosa pieza arquitectónica muy común entre escuelas urbanas y municipales construidas a fines del siglo XIX y durante las primeras décadas del siglo XX. Su patio es particularmente pequeño y cercado por todos sus lados. El ruido del tráfico se oye alrededor del perímetro del patio y el edificio. El vecindario en el que se encuentra la escuela puede ser considerado de clase media. Las casas son estructuras de dos pisos en general, construidas una muy cerca de la otra a lo largo de calles residenciales que se ramifican hacia las autopistas principales de la ciudad.

Cuando uno ingresa a la escuela, se observa un letrero muy discreto que dirige a los visitantes hacia la dirección de la institución educativa. Es difícil encontrar esa oficina, pues es un aula transformada que casi no se distingue de las demás entradas dentro del edificio. Los pasadizos son impecables y 
silenciosos. Los techos son altos; sus grandes ventanas dejan entrar la luz natural y el aire es fresco. El espacio es iluminado, amplio, abierto y relajado. Los niños caminan dentro del edificio con tranquilidad y nos sonríen. En las aulas de clase, también, hay espacios de trabajo activos aunque tranquilos, donde los niños parecen deambular trabajando en diversos tipos de proyectos. El edificio mismo no es nuevo, pero definitivamente lo mantienen y cuidan bien.

Dentro del aula a la que ingresamos, observamos que los niños trabajan en grupos problemas de matemáticas reunidos en "centros». Cada centro está compuesto de una mesa con un juego de materiales diseñados para ayudar a reforzar los conceptos. A los alumnos se les entrega un problema en una tarjeta de color brillante y en cada estación los niños trabajan en grupo para resolver la tarea que se les asignó. Los alumnos se formulan preguntas entre sí, ofrecen ayuda y sugerencias, registran sus resultados; ocasionalmente, hacen una broma o un comentario divertido, pero en general la atmósfera es bastante productiva. En un inicio, es difícil encontrar al maestro, pero luego lo encontramos encorvado en un escritorio trabajando con unos alumnos en una de las estaciones.

Cuando la clase de matemáticas ha concluido, los niños sin hacer ruido reúnen los materiales en las estaciones y los devuelven a un carrito portable y regresan a sus asientos. Un niño lleva el carrito y devuelve los materiales al rincón del salón donde se guardan los materiales de matemáticas en tarros etiquetados y estantes. Notamos que cada curso del programa curricular tiene su propia sección en el aula. La sala tiene una biblioteca muy bien surtida en un rincón dedicado a la lectura. En este rincón, hay un sillón y una silla acolchada. Otra área del salón contiene materiales de ciencia y otro rincón del salón alberga los violines de los niños. Todas las paredes están decoradas con trabajos de los niños, así como con pósteres educativos que describen y detallan los principios de aprendizaje y lemas éticos inspiradores para los niños. Hay muchos trabajos publicados de los niños (especialmente, de redacción y arte).

Una vez que los niños han regresado a sus asientos, el maestro asume el papel de un maestro más tradicional y dirige una discusión del tema de matemáticas (en este caso, probabilidad y muestreo estadísticos) que se relaciona con las preguntas que los niños resolvían en los centros. Luego de la discusión, la clase termina para continuar con el almuerzo. Los niños discretamente salen del aula conversando en parejas o pequeños grupos.

La sensación general que uno tiene de esta aula es de urbanidad y orden. Los niños parecen limpios, ordenados y formales en sus intervenciones orales y movimientos físicos. Se desenvuelven seriamente entre sí en grupos 
pequeños y parejas, y lo mismo con su maestro durante las discusiones formales y grupales. Alrededor del aula, se mueven con calma y cuidado. Cada niño tiene su propio espacio para guardar sus útiles y realizar sus trabajos de manera independiente; estos espacios son respetados y bien cuidados. En el patio de recreo, los niños se vuelven físicos y gran parte de la actividad de los niños mayores se organiza en juegos formales de pelota y otras formas coordinadas de juegos. Los niños más pequeños se dispersan en un patio distinto y sus juegos son más bien libres.

Cuando los padres van por sus niños, muchos llegan en autos último modelo. Tanto los padres como las madres entran a la escuela o llegan hasta los alrededores. Estos padres conversan con facilidad con los maestros y, de hecho, se parecen mucho a ellos en su postura, cuidado personal, vestimenta y apariencia física. Es común ver a los hombres vestidos en trajes.

\section{Caos controlado}

Nuestra segunda escuela se encuentra en una comunidad periférica. Es una escuela primaria relativamente grande -según los estándares de la provincia- y alberga a aproximadamente doscientos niños en edad de escuela primaria y sus familias. Esta escuela fue construida en la década de los ochenta y su exterior es de ladrillos. La comunidad en la que se encuentra la escuela está compuesta de viviendas antiguas y pequeñas de madera, así como subdivisiones de residencias más modernas al estilo de «búngalos». Algunas de estas casas acaban de ser pintadas, pero muchas no. Hay unos cuantos árboles a lo largo de las calles que rodean a la escuela y las casas están unidas sobre lotes muy pequeños. La impresión general es que la escuela trabaja para una comunidad económicamente desfavorecida según los estándares provinciales y nacionales, aunque según los parámetros de una gran parte de Nueva Escocia rural parece normal.

Hay una marcada diferencia entre la construcción de la escuela y las otras construcciones de alrededor. En comparación com los edificios del área, la escuela parece imponente, bien construida y relativamente nueva y bien mantenida, especialmente a la distancia. Ubicada sobre una colina y rodeada por amplias áreas recreativas, la escuela parece una rareza física y arquitectónica en sus alrededores. El área donde se encuentra la escuela es mayormente residencial y en esencia no hay evidencia de actividad comercial cerca de la escuela. Las casas son modestas y se encuentran en calles residenciales sin aceras.

La entrada a la escuela contiene varias oficinas acristaladas desde donde las secretarias y el personal administrativo pueden supervisar el punto de 
entrada y salida de la escuela. Obviamente hay niños en las oficinas y se ve a varios de ellos en los pasillos cerca de las puertas de las aulas a pesar de que las clases ya han comenzado. Al pasar por las aulas algunos niños salen a saludarnos o solo mirarnos. Este no es un lugar donde se vean visitantes adultos a diario. Una larga y angosta ventana construida de vidrio mallado bordea la puerta de cada aula. Algunas puertas están abiertas, pero muchas se mantienen cerradas. Algunas de estas ventanas están cubiertas con papel oscureciendo la vista del aula desde el pasillo y viceversa.

El aula al que ingresamos es ordenada pero muy modesta en cuanto a sus materiales de aprendizaje y muebles. Los alumnos se sientan en pupitres individuales y el maestro presenta los materiales desde la parte delantera del aula. Siguiendo las presentaciones del maestro, los alumnos normalmente trabajan de manera independiente en sus pupitres u ocasionalmente en parejas o pequeños grupos. El aula está medianamente decorada con pósteres inspiradores, en su mayoría auspiciados por empresas o por el Estado. Hay un póster que describe la inverosímil imagen de un jugador de hockey de pie cubierto con todos sus implementos de protección leyendo un libro. El aula tiene varias repisas con una serie de libros escolares y un estante de revistas con libros de bolsillo. En una esquina del aula, se encuentra el escritorio del maestro, un librero y sus artículos personales.

Los niños de esta escuela parecen funcionar de dos formas principalmente: activos -con mucha energía- y pasivos. Durante las presentaciones del maestro y cuando están trabajando en el aula, los alumnos son mayormente pasivos. El lenguaje corporal de los alumnos varía de un estado de mucha dedicación a la indiferencia total. Muchos alumnos se retuercen en sus asientos y miran a todos lados. Intervienen o participan durante las presentaciones del maestro y discusiones de la clase con poca frecuencia y, cuando lo hacen, la mayoría de sus respuestas son más bien muy cortas. Los alumnos no parecen entablar conversaciones con los maestros, pero sí ofrecen respuestas verbales a las preguntas a modo de un llenado de espacios en blanco. Los maestros dictan su clase y formulan preguntas que, cuando son respondidas, se hace con frases cortas o con una sola palabra. Nadie parece esperar más. De hecho, gran parte del trabajo que los alumnos realizan en clase es así. Desde sus asientos, los niños leen extractos y responden a las preguntas en oraciones muy cortas. Los alumnos guardan garabatos, archivadores, útiles, así como bocadillos y otras cosas dentro de sus escritorios de metal.

La sensación general que uno percibe en este espacio es la de un caos controlado. Los alumnos parecen ser más o menos mesurados y, de hecho, la escuela es conocida por su sólida disciplina, su cuerpo militar de cadetes y sus logros deportivos. Los pasadizos casi no tienen decoraciones hechas por 
los alumnos (aunque son evidentes los pósteres decorativos comerciales y educativos) y no hay trabajos de alumnos publicados en el aula o las áreas comunes. Además, existe un estante con trofeos deportivos a la entrada de la escuela. Los alumnos se muestran tímidos con los extraños e incluso con sus maestros y muchos de ellos no se esfuerzan tanto para conversar con los adultos. En realidad, responden de manera mínima a la interacción con adultos.

Cuando muchos alumnos se desplazan en el aula, sus movimientos físicos tienden a ser algo descontrolados, como si hubiesen sido liberados después de haber estado en cautiverio. Muchos niños imitan las poses comunes de los héroes de acción y atletas profesionales -luchadores, especialmentey copian sus movimientos agresivos. A menudo, estos gestos los hacen en parejas con otro niño al imitar el combate de uno contra uno. Los movimientos de los niños son rápidos y bruscos y las conversaciones de los varones en particular son en gran parte monosilábicas. Las niñas tienden a formar pequeños grupos y conversar entre ellas de forma animada, a menudo estallando en carcajadas y llamando a gritos a otros individuos o grupos. Estos patrones generalmente se reproducen en el patio de juegos y en el bus, o al caminar a casa o la escuela.

Hay muy poca evidencia de la presencia paterna en la escuela. Muchos niños caminan a casa de la escuela a la hora del almuerzo sin la compañía de un adulto. Cuando se ve a padres en la escuela, casi siempre se trata de un miembro familiar femenino. Durante mi visita a la escuela, no observé conversaciones prolongadas o lo que podría considerarse como conversaciones «amigables» entre los padres y el personal. Los padres normalmente esperan a que sus hijos salgan del edificio en los autos estacionados en la calle adyacente a la escuela.

\section{Una gran familia}

Nuestra tercera escuela se encuentra en una comunidad rural. Es una estructura de madera relativamente pequeña, construida a fines de los años 50 como parte de un programa de fusión de escuelas rurales. La escuela está sobre una colina en un pequeño pueblo que históricamente depende de pequeñas granjas y la silvicultura como infraestructura económica. Puesto que estas industrias han sido "reestructuradas» por la concentración de capitales y las políticas de Estado que no apoyaron a los pequeños productores, la inscripción en las escuelas ha caído tremendamente en las últimas dos décadas. A pesar de su cuerpo estudiantil relativamente reducido de menos de 100 alumnos, alberga a niños de un área geográfica de unos 35 kilómetros. 
La escuela originalmente fue diseñada para albergar a una población mucho mayor; por ello, la sensación que se percibe dentro del edificio es de considerable espacio.

El estacionamiento de la escuela a menudo está lleno de autos de padres voluntarios, que llegan a la escuela para participar en el programa de almuerzos y visitar a sus hijos en las aulas. Es evidente que la escuela es considerada como propiedad de la comunidad. El edificio es usado por las tardes y después de horas de clase para una variedad de programas comunitarios. Aparentemente, la escuela es arquitectónicamente consistente con las demás construcciones vecinas, que en su mayoría son casas bien mantenidas del siglo XIX e inicios del siglo XX, mezcladas con estructuras más modernas tipo búngalos. A pesar de la despoblación y la reestructuración industrial, la comunidad sigue dependiendo económicamente de una industria de recursos primarios. Esto se evidencia en el equipamiento y las construcciones anexas cercanas a muchas viviendas. Las camionetas son los vehículos comunes.

En el ingreso y el vestíbulo de la escuela, hay fotografías de los niños participando en una variedad de actividades dentro y alrededor de la escuela. La presencia de adultos en las fotografías transmite la impresión de una continua presencia de adultos en los eventos y las actividades escolares en los que participan los niños. En el vestíbulo, un grupo de tímidos niños, que parecen sentirse cómodos alrededor de adultos, nos dan la bienvenida. Al igual que en la segunda escuela, los niños parecen animados e incluso sus movimientos son frenéticos. Ocasionalmente, aparecen niños pequeños revoloteando y golpeándose unos con otros a medida que van saliendo del edificio hacia el patio de juegos.

La escuela parece esforzarse mucho para transmitir la impresión de ser una institución central de la comunidad, o más aún, el «corazón de la comunidad». Esto es más que un mito, pues durante generaciones muchas reuniones importantes de la comunidad se han realizado en la escuela. Cuando la escuela ha sido amenazada de cierre (justificado por un menor registro de alumnos) -como muchas veces ha sucedido durante las últimas dos décadas-, los miembros de la comunidad han emprendido campañas sobre la importancia de la escuela para la comunidad. El término "comunidad», en un sentido importante, es definido por la geografía del área de alcance de la escuela, y su consolidada escuela primaria es el resultado de la fusión de una docena de escuelas de pueblo que funcionaron hasta finales de los años 50 cuando el sistema educativo provincial «se modernizó». De hecho, el lenguaje de la comunidad es preponderante y explícitamente mencionado en el papel membretado de la misma y en todos los materiales impresos en todo 
el edificio. Detrás de sus paredes, los adultos se mezclan con los niños. Hay supervisores en el patio de juegos y señoras encargadas del almuerzo que conocen a todos los niños (y padres) por su nombre. Todos en la escuela parecen llamarse por su nombre. La mayoría de adultos en la escuela son mujeres y cuando llegan hombres a la escuela, a menudo, parecen incómodos. La escuela es, en gran parte, un lugar de mujeres en esta comunidad. El programa curricular combina el aprendizaje tradicional mediante libros, que en parte se desarrolla en sesiones de aprendizaje grupales como en las otras escuelas. También, hay evidencia de técnicas basadas en proyectos más activos y participación de la comunidad, una activa área de producción de arte, un centro comunitario de tecnología de la información y espacios tipo talleres en toda la escuela.

La atmósfera que se percibe es feliz y activa, mas no es un lugar del que alguien pueda concluir que existen altos estándares académicos. Muchos de los niños luchan con las matemáticas, la lectura y la redacción. Si bien los resultados estándares de aprendizaje y matemáticas pueden ser un poco mejores que en la escuela anterior, están muy por debajo de los de la primera escuela. Muchos de los alumnos afirman que les gusta trabajar con sus manos. Al escuchar el inglés no estándar que los alumnos hablan, quizás, no sea tan difícil entender por qué, como grupo, luchan en los cursos de idiomas (y ¿qué cursos no son cursos de idiomas?). Los maestros con frecuencia pasan muchísimo tiempo con ciertos niños trabajando para «nivelarlos a su grado», pero muchos de los maestros entienden que el resultado de la prueba será de un promedio bajo para la mayoría de los alumnos.

\section{¿Qué podemos hacer con estos datos?}

Este tipo de detalles que acabo de relatar generalmente no son los que aparecen en los análisis de las escuelas y la educación. Los datos que he usado para crear el reporte anterior son ciertamente anecdóticos e incluso quizás impresionistas. Representan el tipo de conocimiento local que es sentido común para cualquiera que trabaje en la escuela o la visite con regularidad como padre. Contienen sugerencias implícitas sobre cómo pueden mejorar cada una de estas escuelas y cómo cada una de ellas representa diferentes lugares en la diversa geografía social de Canadá. Existen miles de otros reportes que pueden crearse en otras localidades. Este es el nivel de datos que se necesita para mejorar sustancialmente las escuelas y que nunca se podrán generar a través de ejercicios de recolección descendente de datos como las pruebas estandarizadas internacionales. Más importante todavía, cada una de estas 
escuelas representa una geografía social, cultural, económica y política que se ha desarrollado en diferentes comunidades, puesto que estos lugares han sido transformados de distinta manera por el desarrollo capitalista.

El reportaje descendente de las pruebas estandarizadas y las evaluaciones internacionales de habilidades es problemático en una región en la que los resultados son subestándares (como siempre lo son en las provincias atlánticas de Canadá). La solución tiende a ser articulada en términos de cómo lograr que las localidades de "bajo rendimiento» sean parte del sistema con el fin de obtener mejores resultados en las pruebas estandarizadas. Aunque el objetivo de las pruebas nunca fue dar forma o influir en la práctica, enseñar a los mejores se ha convertido en una norma para escuelas, distritos, provincias y naciones que luchan por disciplinar a sus maestros y alumnos para mejorar su rendimiento en tareas genéricas de evaluación urbano céntricas. En otras palabras, el programa curricular se uniformiza esencialmente a través del mecanismo de evaluación estandarizada de alta inversión.

Los datos que he reportado anteriormente son más matizados que los reportes estadísticos normalmente presentados en los reportes educativos. Estos reflejan el lugar y una perspectiva ascendente (de abajo hacia arriba). Se resisten a las correcciones y argumentos sobre cómo funciona una mayor estandarización, como una marea creciente que supuestamente hace flotar a todos los barcos. Estas anécdotas ilustran cómo el prolongado proyecto histórico de la educación pública y los estándares comunes nunca han homogeneizado a las escuelas en términos de los resultados académicos que producen. Este es el caso incluso en un país relativamente desarrollado o capitalista avanzado, que es además reconocido por los estándares internacionales por sus altos niveles de equidad y sólidos programas sociales. No debemos esperar que sea así. Cuando comparamos los resultados de las tres escuelas en las evaluaciones estandarizadas provinciales de alfabetización y matemáticas, aparece una imagen interesante. En la escuela urbana de clase media, como era de esperar, todos los resultados son altos en 3er grado (8-9 años) y 6to grado (11-12 años). En la escuela suburbana de la clase trabajadora y en las escuelas de comunidades rurales, los resultados son previsiblemente más bajos que en la escuela del vecindario de clase media. Al mismo tiempo, en las últimas dos escuelas, los resultados de las pruebas en realidad aumentan del 3er al 6to grado. El aumento del 3er al 6to grado es más alto en la escuela de la comunidad rural. Esto sugiere que en estas comunidades, en las que el capital cultural paternal es "más bajo», de hecho, la educación puede hacer una importante diferencia en las escuelas pequeñas y centradas en la comunidad. En otras palabras, aunque los resultados sean más bajos, estas escuelas pueden ser tan o incluso más "efectivas» que las escuelas de 
alto rendimiento de la clase media. La única forma de saber esto es combinando el análisis estandarizado cuantitativo de alto nivel del rendimiento de la escuela con un análisis más localizado de ciertas escuelas y los recursos económicos, culturales y sociales a los que las familias de esas comunidades tienen acceso.

A medida que las geografías y discursos globalizados educativos han llegado a dominar las teorías políticas y la práctica, las investigaciones educativas cualitativas, matizadas y orientadas a los lugares están siendo llevadas a los márgenes. Como Patti Lather (2004) y otros han demostrado, el giro impulsado por la ideología hacia el análisis «científico» y «basado en la evidencia» del rendimiento educativo ha marginado estudios cualitativos de lo que está sucediendo dentro de las escuelas y otros centros educativos. En un trabajo anterior, he denominado a esto el edumómetro, una continua búsqueda de un instrumento de medición educativa neutral como un termómetro que mide la temperatura o un velocímetro que mide la velocidad (Corbett, 2008). Estos tipos de estudios han demostrado de manera consistente que las características sociales y personales de la gran mayoría de quienes ingresan a la caja negra de la educación como niños pequeños no terminan muy diferentes en términos de oportunidades de vida que los hombres y mujeres jóvenes en los que se convierten y que emergen al final del proceso. Al mismo tiempo, en aquellos casos en los que las escuelas se las arreglan para transformar vidas, aprendemos menos de lo que podríamos sobre los detalles caóticos, ascendentes y locales de cómo es que sucede.

La pregunta que el macroanálisis efectivamente nunca puede responder es por qué sucede esto y cómo se logra. ¿Cómo las escuelas logran canalizar a chicos de la clase trabajadora en empleos de la clase trabajadora, como Paul Willis (1981) manifestó hace más de 30 años? O, ¿cómo se quiebra el ciclo y efectivamente la hegemonía (Gramsci, 1971; Orlowski, 2011) resiste su interpelación (Althusser, 1970)? Dicho de otra manera, ¿cómo se logra la violencia simbólica descrita por Bourdieu (1984)? ¿Cómo se convence a los grupos económicamente desfavorecidos, racializados y marginalizados que ellos merecen tener ese destino en los sistemas educativos? Esta es la compleja historia de cada lugar que difiere entre una escuela y otra, de lugares urbanos a rurales en los que las prácticas y estructuras sociales, culturales y de empleo difieren tremendamente. Justo cuando comenzábamos a saber qué preguntas formular sobre cómo desarrollar la educación inclusiva y equitativa, existe el peligro en Canadá respecto a que gran parte de los esfuerzos en el proyecto educativo pasarán ahora por mediciones y vigilancia con el fin de mejorar las calificaciones de las pruebas. Además, por supuesto, existe el debate sobre cómo los mejores resultados se traducen en mejores oportu- 
nidades de vida para niños marginalizados. De hecho, este es un tema que merece una investigación más exhaustiva, pues no está del todo claro.

Desde mi perspectiva, entonces, necesitamos saber más sobre los lugares que básicamente son reducidos a puntos de datos en un análisis educativo descendente. Sin embargo, esto no significa que las microgeografías están de algún modo separadas de las configuraciones espaciales más amplias o que las escuelas individuales son lo único que debemos comprender. Macro, meso y micro son diferentes escalas en las que se pueden analizar los mismos problemas (Nespor, 2004); y, de hecho, la dependencia exclusiva en el análisis ascendente o descendente del fenómeno educativo se ha justificado a la fecha. Es también importante recordar que todos los lugares están en constante movimiento en la actualidad y contienen lo que Massey llama el carácter de «mezcla». Respecto a ello, Massey escribe lo siguiente:

No se pueden asumir la coherencia preestablecida, la comunidad o la identidad colectiva. Por el contrario, la combinación de lugares exige cierta negociación. En un marcado contraste con una perspectiva del lugar como algo establecido y preconcebido, con una coherencia que solo podría verse afectada por fuerzas externas, los lugares como aquí los presentamos de cierta forma exigen intervención; representan un reto (2005, p. 141).

La dinámica del cambio y la transformación de los lugares son una característica crónica de la producción social, por la que actualmente estamos luchando para hacerla valer en nuestro análisis de las frecuentes categorías estáticas como clase social, raza, género y por supuesto educación. De hecho, mi propio hogar rural está cambiando rápidamente y volviéndose muy diverso. Si la generación anterior de investigación social nos ha enseñado algo, es que todo está en movimiento, incluyendo las herramientas estructurales que hemos usado para pensar sobre el hecho de cómo estamos juntos.

Emprender el reto creativo de negociar una solución es una problemática de política espacial, o lo que Edward Soja (2010) Ilama «justicia espacial». Es en el área de las comunidades cambiantes que las escuelas trabajan y donde las personas escogen rendirse o emprender un reto, exigir acceso a espacios cercados o exclusivos y movilidad. Yo propongo que el objetivo fundamental de las escuelas sea el territorio abierto a la imaginación e improvisación (Corbett, 2013). Las interpretaciones contemporáneas del espacio en la educación (Green y Letts, 2007; Leander, Phillips y Taylor, 2010) plantean nuevas preguntas sobre la forma como las geografías desde un aula de clases hasta la escala global son plásticas y cambiables, pero al mismo tiempo diferentes. En el centro de las ciudades y en comunidades rurales remotas, las evaluaciones comercializadas a gran escala pueden servir para promover 
cambios positivos o -en su defecto- para sembrar las semillas de la paranoia y el desprecio por uno mismo, que a cambio tienden a certificar y reproducir una subclase multigeneracional. En los Estados Unidos, la reorientación de los sistemas estatales casi exclusivamente hacia una mejora del rendimiento de las pruebas estandarizadas descendentes ha llevado a la privatización, la cuasi privatización, la desprofesionalización de la enseñanza, la instrucción programada y el quiebre del gran sindicato de maestros (Hacker y Dreifus, 2013). Los intentos por restringir y cerrar las posibilidades innovadoras de los espacios educativos son el rostro de la dominación que, a mi parecer, debe llamar a los educadores progresistas y radicales a la resistencia.

Como bien señala Massey, "para que el futuro sea abierto, los espacios también deben serlo» (2005: 12). El cierre del espacio hoy se logra mediante la amplia y extensa comercialización de las prácticas y evaluaciones educativas que claman por un debate. Necesitamos nuevas geografías educativas para contrarrestar la violencia simbólica que se normaliza en los esquemas educativos descendentes independientemente de las buenas intenciones. Asimismo, me parece que es importante que los investigadores educativos (especialmente, aquellos que trabajan cualitativamente en escuelas locales) conecten sus trabajos con debates políticos, económicos, culturales y sociales más amplios sobre retos actuales dentro del capitalismo global. La estructura de clases y la geografía de las sociedades capitalistas avanzadas yacen sobre una base incierta de mercados estables, energía barata, crédito fácil y bienes, servicios y entretenimiento producidos en masa. Estos elementos, vistos en conjunto, han creado condiciones ambientales tremendamente precarias, las cuales han hecho que muchos de los más profundos artistas y críticos sociales del mundo imaginen mundos distópicos en el futuro. En realidad, es aquel pobre global y aquellos en los márgenes de las sociedades ricas-como los pueblos aborígenes en el norte de Canadá, la clase trabajadora rural desfavorecida y los centros de las ciudades- quienes luchan para sobrevivir en medio de una situación de desempleo galopante en un mercado inflado de viviendas urbanas.

Lo que finalmente deseo aclarar es que las discusiones sobre justicia social que son descendentes, descontextualizadas, carentes de análisis cultural y espacial no tendrán sentido respecto a lo que justicia social verdaderamente significa y lo que en términos de las políticas educativas provienen de estas interpretaciones. Necesitamos implementar una compleja política educativa de escala múltiple y poner en práctica discursos en los que el espacio y el lugar sean una parte integral del pensamiento del cambio educativo. En esto, radica para mí la importancia actual de la investigación intensa, matizada, teóricamente sofisticada, exigente, cualitativa, orientada a lugares especí- 
ficos sobre la educación además del análisis a gran escala del rendimiento educativo.

\section{Referencias}

Althusser, L. (1970). Ideology and Ideological State Apparatuses. En Althusser, L., Lenin and Philosophy and other Essays. New York: Monthly Review Press, 121-76, 160.

Appadurai, A. (1996). Modernity Al Large: Cultural Dimensions of Globalization. Minneapolis: University of Minnesota Press.

Ball, S. J. (2012). Global Education Inc.: New Policy Networks and the Neoliberal Imaginary. New York: Taylor \& Francis Group.

Bourdieu, P. (1984). Distinction: A Social Critique of the Judgment of Taste. Cambridge: Harvard University Press.

Castells, M. (2000). The Rise of the Network Society: The Information Age: Economy, Society, and Culture, Volumen I. Londres: Wiley-Blackwell. (2009). The Power of Identity: The Information Age: Economy, Society, and Culture, Volume II (2a. ed.). Malden MA: Wiley-Blackwell.

Corbett, M. (2008). The Edumometer: The commodification of learning from Galton to the PISA. Journal for Critical Education Policy Studies, 6 (1), 1.

Giddens, A. (1979). Central Problems in Social Theory: Action, Structure and Contradictions in Social Analysis. Berkeley: University of California Press. (1990). The Consequences of Modernity. Stanford: Stanford University Press.

Gramsci, A. (1971). Selections from the Prison Notebooks of Antonio Gramsci. (G.N. Smith y Q. Hoare, Traduc.). New York: International Publishers.

Green, B. y Letts, W. (2007). Space, Equity and Rural Education: A Trialectical Account. En Gulson, K. N., y Symes, C. (Eds.), Spatial Theories of Education: Policy and Geography Matters. Routledge.

Hacker, A. y Dreifus, C. (2013, June 8). The Common Core: Who's Minding the Schools? The New York Times. Recuperado de http://www.nytimes. com/2013/06/09/opinion/sunday/the-common-core-whos-mindingthe-schools.html

Halpin, D. y Troyna, B. (1995). The Politics of Education Policy Borrowing. Comparative Education, 31(3), 303-310.

Kovacs, P. (2011). (Ed.). The Gates Foundation and the Future of U.S. "Public» Schools. New York: Taylor \& Francis. 
Lather, P. (2004). This is Your Father's Paradigm: Government Intrusion and the Case of Qualitative Research in Education. Qualitative Inquiry, 10(1), $15-34$.

Leander, K. M., Phillips, N. C. y Taylor, K. H. (2010). The Changing Social Spaces of Learning: Mapping New Mobilities. Review of Research in Education, 34(1), 329-394. doi:10.3102/0091732X09358129

Lingard, B. (2010). Policy borrowing, policy learning: testing times in Australian schooling. Critical Studies in Education, 51(2), 129-147.

Luke, A. (2011). Generalizing Across Borders: Policy and the Limits of Educational Science. Educational Researcher, 40(8), 367-377.

Martino, W. y Rezai-Rashti, G. (2013 in press). «Gap talk» and the global rescaling of educational accountability in Canada. Journal of Education Policy.

Massey, D. B. (2005). For Space (1a. ed.). Londres: Sage Publications Ltd.

Meyer, H. D. y Benavot, A. (Eds.) (2013). PISA, Power, and Policy: the emergence of global educational governance. Oxford: Symposium Books.

Milner, H. R. (2013). Rethinking Achievement Gap Talk in Urban Education. Urban Education, 48(1), 3-8.

OECD (2011). Strong Performers and Successful Reformers in Education. Lessons from PISA for the United States. OECD Publishing. Recuperado de http://www.oecd.org/pisa/46623978.pdf

Orlowski, P. (2011). Teaching About Hegemony. Heidelberg, Londres and New York: Springer.

Rizvi, F. y Lingard, B. (2010). Globalizing Education Policy. Londres: Taylor \& Francis.

Rose, N. (1999). Governing the Soul: The Shaping of the Private Self (2a. ed.). Londres: Free Association Books.

Soja, E. W. (2010). Seeking Spatial Justice. Minnesota: University of Minnesota Press.

Spring, J. (2008). Globalization of Education: An Introduction (1a. ed.). New York: Routledge.

Willis, P. (1981). Learning to Labor: How Working Class Kids Get Working Class Jobs (Morningside). New York: Columbia University Press. 